\title{
Efficient and Reliable Solar Panels for Small CubeSat Picosatellites
}

\author{
Ivo Vertat ${ }^{1}$ and Ales Vobornik ${ }^{2}$ \\ ${ }^{1}$ Department of Applied Electronics and Telecommunications, University of West Bohemia, 30614 Pilsen, Czech Republic \\ ${ }^{2}$ Department of Technologies and Measurement, University of West Bohemia, 30614 Pilsen, Czech Republic
}

Correspondence should be addressed to Ivo Vertat; ivertat@kae.zcu.cz

Received 30 October 2013; Accepted 16 March 2014; Published 25 June 2014

Academic Editor: Mahmoud M. El-Nahass

Copyright (C) 2014 I. Vertat and A. Vobornik. This is an open access article distributed under the Creative Commons Attribution License, which permits unrestricted use, distribution, and reproduction in any medium, provided the original work is properly cited.

\begin{abstract}
CubeSat picosatellites have a limited area of walls for solar cells assembling and the available area has to be effectively shared with other parts, such as planar antennas, optical sensors, camera lens, and access port. With standard size of solar cell strings, it is not possible to construct a reliable solar panel for CubeSat with redundant strings interconnection. Typical solar panels for CubeSat consist of two solar cell strings serially wired with no redundancy in case of solar string failure. The loss of electric energy from one solar panel can cause a serious problem for most picosatellites due to minimum margin in the blueprints of the picosatellite subsystem power budget. In this paper, we propose a new architecture of solar panels for PilsenCUBE CubeSat with a high level of redundancy in the case of solar string failure or following switched power regulator failure. Our solar panels use a high efficiency triple junction $\mathrm{GaInP}_{2} / \mathrm{GaAs} / \mathrm{Ge}$ in the form of small triangle strings from the Spectrolab Company. A suitable technology for precise solar cell assembling is also discussed, because CubeSat picosatellites are usually developed by small teams with limited access to high-end facilities.
\end{abstract}

\section{Introduction}

At present, there is a great boom for small satellites for new technology demonstration and verification with low cost and short preparation time before final application of the technology on conventional satellites. Standardized CubeSat picosatellites are popular worldwide for these purposes. A basic unit of a CubeSat picosatellite [1] is $100 \mathrm{~mm}$ in length, $100 \mathrm{~mm}$ in width, and $113.5 \mathrm{~mm}$ in height; one picosatellite wall offers an area of solar cells no more than $83 \mathrm{~cm}^{2}$. Such a limited area together with a mass limit of $1.33 \mathrm{~kg}$ (limiting complicated deployable mechanisms) does not provide a high amount of electric energy for a CubeSat power supply. When silicon-based solar cells are used (due to its low cost), it is not possible to predict more than $1 \mathrm{~W}$ of power generation. When more efficient gallium arsenide based multijunction solar cells are used, the amount of generated energy increases up to $3 \mathrm{~W}$ [2]; however, it also comes with a high cost increase. With a limited financial budget of small CubeSat teams, only standardized cell string sizes are used for assembly instead of customized sizes. Only two standard sized cells per one wall are possible to place. These two cells on one wall are wired serially for several practical reasons. The higher output voltage of serial wiring offers higher efficiency of subsequent switched regulators and also the output voltage is better suited to the final voltage of Li-Ion or Li-Pol accumulators which help to enable a safe mode of direct accumulator charging in case of nonstandard situations on picosatellite power buss after system failure. A standard solar panel with two multijunction solar cells can be found in $[3,4]$.

On the other hand, such serially connected solar cells are the source of unreliability in the case of cells damage during carrier launch or during the operational temperature cycling with thermal stress (low temperatures after flight in Earth shadow as well as high temperatures after flight in sunlight are expected). Solar cells can also be broken by micrometeor impact. The total loss of electric energy from one CubeSat wall is a serious complication for the picosatellite operation planning due to a low margin in the subsystem power budget. This weakness of CubeSat picosatellites is one of several 


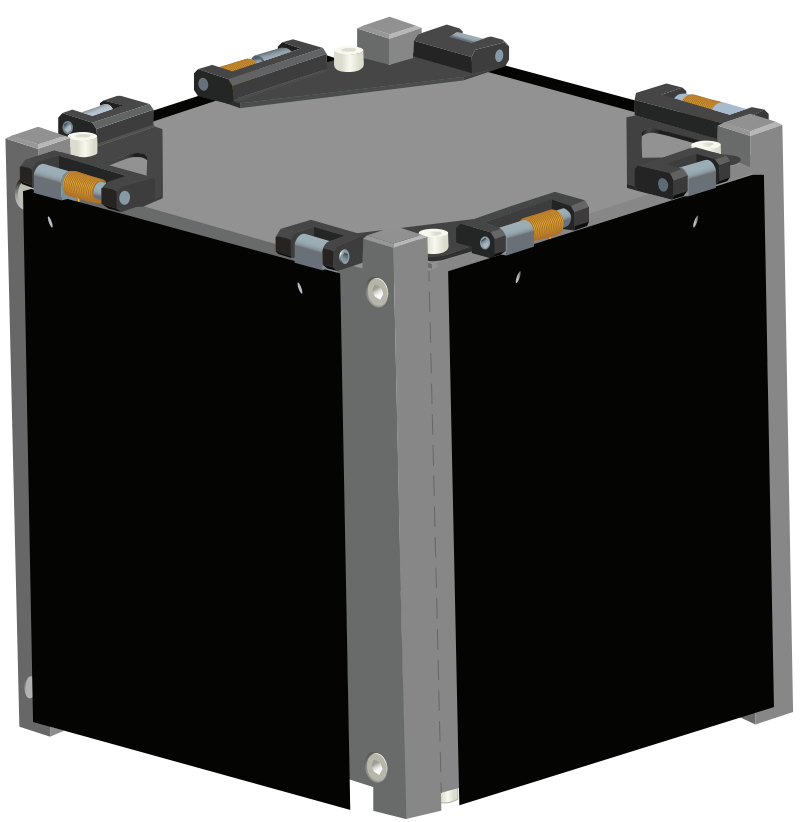

Figure 1: Model of PilsenCUBE picosatellite [2] based on CubeSat standard.

reasons for our research within the PilsenCUBE project and for the developing of our picosatellite (in Figure 1).

Designs of solar panels have to respect many demands. In inland usage the solar cell efficiency and solar panel area are not usually critical demands due to cheap land. However, the unstable meteorological conditions should be critical to solar panels reliability. Solar panels are faced to rain, snow, freeze, hailstone, overheating, sandstorm, and many other effects. In $[5,6]$ the wind-induced pressures on inland solar panels are evaluated. In space usage the solar panels are not faced with the unstable meteorological conditions, but solar panel area and solar cell efficiency are critical due to satellite (or picosatellite) mass limit. The effect of radiation, launch vibration, fast thermal expansion, and so forth must also be considered. In $[7,8]$ the effects of micrometeoroids and space debris impacts on satellite solar panels are described.

In inland generation of the power from sunlight several novel approaches arise. For example the optical concentrators described in [9] allow using much smaller area of the expensive solar cell materials with higher efficiency. Some hybrid technologies use the thermal part of sunlight for the conversion efficiency increase [10, 11]. These technologies are far from practical usage on current satellites, where the research is aimed at novel cell materials with higher efficiency and more precise solar panels pointing toward the Sun.

The PilsenCUBE picosatellite project has three main goals. One of them is to ensure an effective, reliable, and sufficient power supply of CubeSat electronic subsystems, which is briefly described in this paper. Other goals aim at the ensuring of radiation susceptibility and radio connectivity data throughput, which is described in $[12,13]$.

The remainder of this paper is organized as follows. In Section 2, the results of accessible solar energy calculations are presented as well as solar cell properties measurement resumed from our previous work [2]. In Section 3, the concept of efficient and reliable solar panels for CubeSat picosatellites is introduced. Several technological aspects of solar cells assembling are mentioned in Section 4, related to the ease of cells assembling and thermal-vacuum acceptance tests and thermal stress susceptibility. In Section 5, the solar panels arrangement on our PilsenCUBE picosatellite and its simple deployable mechanisms and results of expected power generation are described. All work and the results of solar panel prototype development are concluded in Section 6.

\section{Solar Energy for CubeSat Supplying}

For conventional civil satellites, a solar flux is the only source of energy. Power supplying of CubeSat picosatellites is complicated by the small area for solar cells without difficult and heavy deployable panels. Also, the precise pointing of CubeSat picosatellites and its solar cells towards the Sun is not possible due to the difficulty of carrying out attitude determination and control systems with low mass and low onboard power consumption. As a result, CubeSat picosatellites have a low amount of electric energy.

2.1. Simplified Calculation of Accessible Energy. Most CubeSat projects use a very simplified way of how to calculate the amount of energy generated by solar cells. In such a case, the power generated by solar panel is given as follows:

$$
P=\eta_{n} \cdot S \cdot A \cdot \sin \varphi,
$$

where $\eta_{n}$ [\%] is nominal efficiency of used solar cells, $S\left[\mathrm{~W} / \mathrm{m}^{2}\right]$ is sunlight power density, $A$ is the area of solar cells on panels, and $\varphi\left[{ }^{\circ}\right]$ is the angle of sunlight irradiance measured from the panel base plane. Nominal efficiency is considered as constant without dependencies and its value is obtained from manufacturer documentation, typically measured in laboratory conditions at $25^{\circ} \mathrm{C}$ temperature. In reality, the efficiency is temperature dependent and is irradiance angle dependent due to surface refraction and losses in cover layers. Both dependencies can dramatically influence the real amount of accessible energy from solar cells which will be also shown in this paper.

Sunlight power density is approximately considered as $1350 \mathrm{~W} / \mathrm{m}^{2}$ (value is valid above the atmosphere near the Earth, related to AM0 model of the sunlight spectrum). Solar panels for CubeSat are not assembled by more than $60 \mathrm{~cm}^{2}$ of solar cells due to its standard sizes and the panel area sharing with other necessary components of picosatellite. The minimum of generated power occurs when one of the CubeSat walls is irradiated perpendicularly ( $\varphi$ equal to $90^{\circ}$ ) because in such a case only one solar panel generates the power. The maximum generated power occurs when three walls are irradiated equally ( $\varphi$ equal to $35^{\circ}$ ).

In Figure 2, the result of simplified calculations with the usage of cheap silicon-based solar cells $\left(\eta_{n}\right.$ equal to $\left.12 \%\right)$ and for an arbitrary angle of sunlight irradiance is shown. The results of same calculations with modern gallium arsenide based high efficiency multijunction solar cells $\left(\eta_{n}\right.$ equal to 


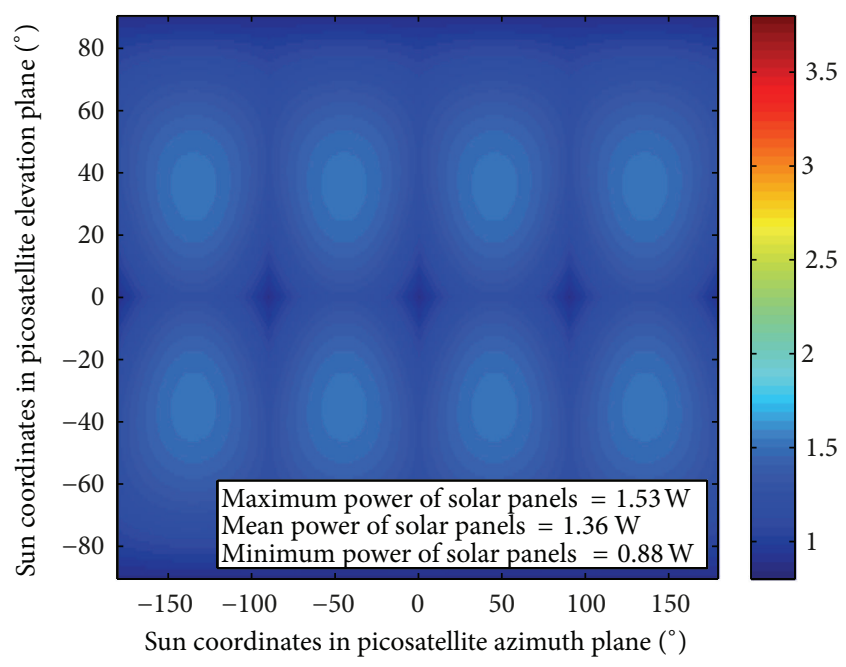

FIGURE 2: Power generation of silicon-based solar panels placed on CubeSat walls in dependence on Sun position.

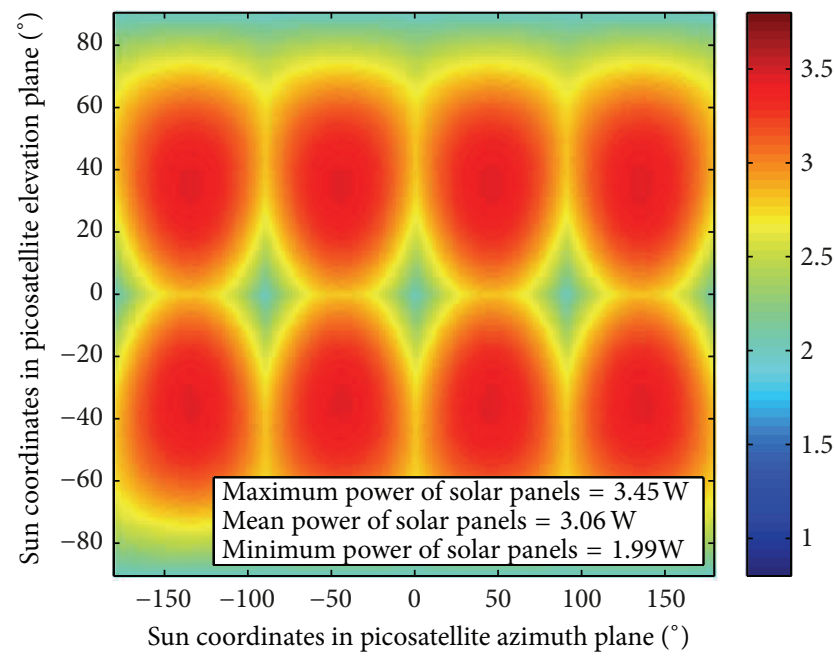

FIGURE 3: Power generation of gallium arsenide based solar panels placed on CubeSat walls in dependence on Sun position.

27\%) are shown in Figure 3. Six solar panels (each with $54.6 \mathrm{~cm}^{2}$ of solar cells) on CubeSat walls are postulated in the calculations.

The mean power of solar panels is calculated as the average value from the graph, so the Sun position has the same probability in all directions (e.g., no attitude control system is working). The usage of gallium arsenide based cells instead of silicon-based cells represents a significant increase of accessible power for the CubeSat picosatellites supply; however, in both cases any efficiency dependencies are disregarded. This method of calculations is not acceptable for proper picosatellite operation planning.

2.2. More Accurate Results of Accessible Energy. Previous methods of accessible power calculation get more positive results in comparison to the reality of orbit conditions. Solar cells on picosatellites operate in a wide range of temperatures.

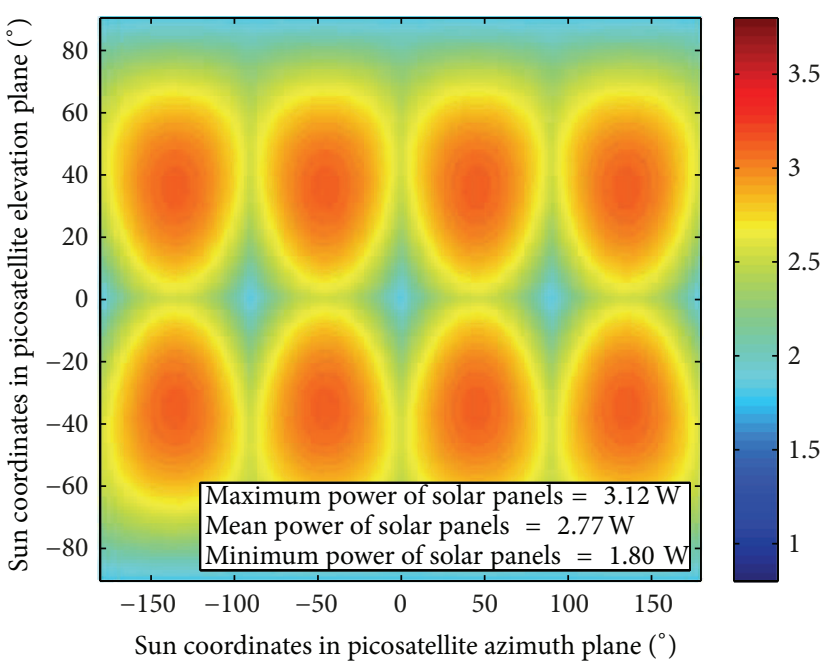

FIgURE 4: Power generation of gallium arsenide based solar panels placed on CubeSat walls-solar cell temperature $60^{\circ} \mathrm{C}$; angle efficiency dependence is not covered in calculations.

After leaving from the Earth shadow, they are deeply frozen and their temperature increases rapidly during operation in sunlight. The highest temperature is limited by heat transfer through picosatellite structure to the shadowed sides and its reradiating in infrared wavelength. On a CubeSat, it is necessary to consider high temperatures because there are no systems for active thermoregulation. The efficiency of solar cells considerably decreases with temperature increase and the real operational temperatures on CubeSat are much higher than the $25^{\circ} \mathrm{C}$ considered in simplified calculations with constant efficiency.

Disregarding of surface reflection and absorption in cover layers is the second source of inaccuracy. When the sunlight irradiates the cells at a low angle, the total reflection can happen for part of sunlight spectra. More wavelengths of sunlight spectra can totally reflect from the surface at lower angles of irradiance and do not contribute to power generation. Also, with a lower angle, the line of sight through cover layers is prolonged and losses are increased. This decreases the solar cells efficiency at lower irradiance angles.

We presented the results of temperature dependence of efficiency and also the results of irradiance angle dependence in [2]. These tests were performed on TASC solar cells from the Spectrolab Company, which are used for our novel solar panel design for the PilsenCUBE project. For illustration, the temperature increase from $25^{\circ} \mathrm{C}$ to $60^{\circ} \mathrm{C}$ means that the efficiency decreased from nominal value $27 \%$ to $24.4 \%$. Also, cells irradiated from a $35^{\circ}$ angle (case of optimal CubeSat irradiation) have decreased efficiency from nominal $27 \%$ to $22.8 \%$. With both effect joint influences (temperature $60^{\circ} \mathrm{C}$ and irradiation angle $35^{\circ}$ ), the efficiency of cells falls down to $20.6 \%$. More precise results of accessible power are shown in Figures 4, 5, and 6, where these measured properties of TASC solar cells are covered. In comparison with Figure 3, it represents the dramatical decrease of accessible power from solar cells. 


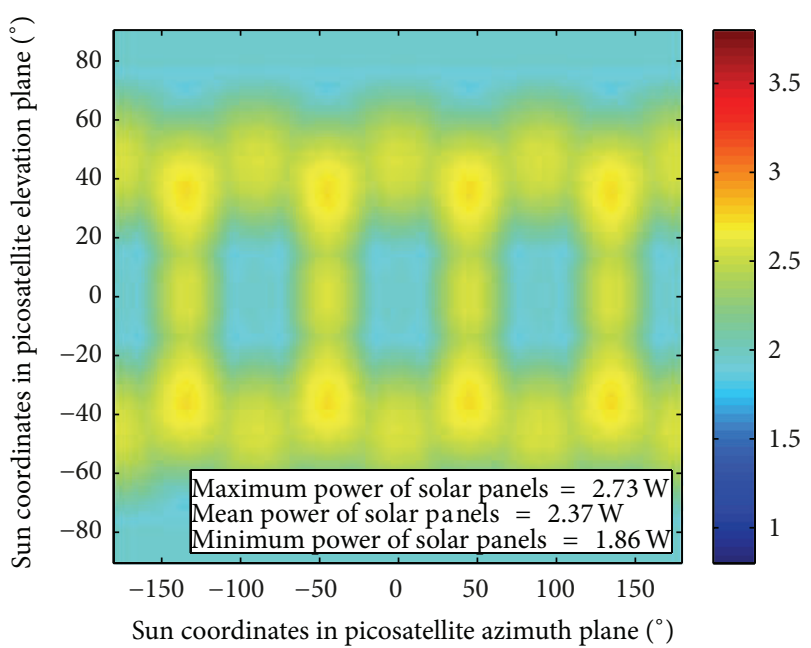

FIGURE 5: Power generation of gallium arsenide based solar panels placed on CubeSat walls-angle efficiency dependence is covered, $25^{\circ} \mathrm{C}$ default temperature of solar cell.

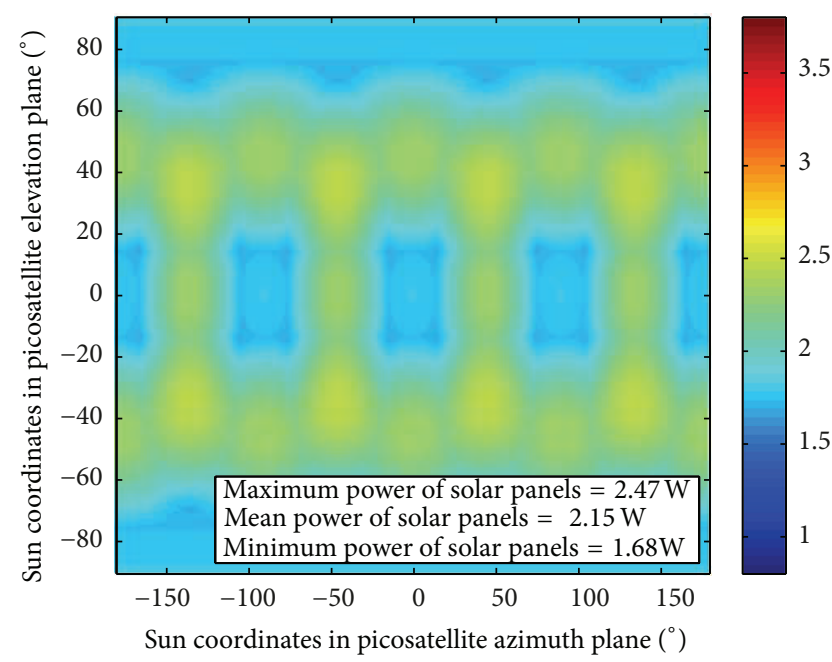

FIgURE 6: Power generation of gallium arsenide based solar panels placed on CubeSat walls-solar cell temperature $60^{\circ} \mathrm{C}$; angle efficiency dependence is also covered in calculations.

All tests of solar cells were realized with the halogen lamp light source with the optical filters to increase color temperature close to the value of sunlight above the atmosphere. This light source was chosen due to continuous light spectrum without strong parasite peaks and due to low budget of all picosatellite development. However, the potential usage of professional and price expensive sun light simulators (based on xenon arc lamp, metal-halide arc lamp, or in combination with halogen lamp) cannot provide more accurate results due to strong parasitic peaks in spectrum.

The amount of accessible power from the CubeSat solar panels with covered efficiency dependencies is relatively low even in the case of gallium arsenide solar cells. Moreover, the generated amount of power will continuously decrease with radiation degradation of the solar cells operated in orbit conditions and decrease in steps with cells destroyed by micrometeors or switched regulator failure. Our novel design of CubeSat solar panels, including deployable mechanisms, should improve the performance and reliability of the CubeSat power supply.

\section{Novel Design of CubeSat Solar Panel}

Several years ago the Spectrolab Company released its small triangle TASC solar cells from $\mathrm{GaInP}_{2} / \mathrm{GaAs} / \mathrm{Ge}$ material and it opened the way for a new advanced architecture of CubeSat solar panels. Also, their low price is suited for small teams and small projects. The small area of TASC cells allows for assembly of a solar panel with high number of cells. A high number of cells per one panel can be used for more advanced interconnection of them into several independence branches to avoid power generation failure from all cells on the same panel.

A basic unit of designed solar panel consists of two serially connected TASC solar cells with total active area $4.55 \mathrm{~cm}^{2}$. An unloaded basic unit has output voltage equal to $5.04 \mathrm{~V}$ and an optimally loaded (in maximum power point of current-voltage characteristic) unit has an output voltage of approximately $4.4 \mathrm{~V}$, perfectly suited for an emergency direct charging of CubeSat accumulators. One basic unit is able to generate $0.166 \mathrm{~W}$ of output power with nominal cell efficiency $27 \%$ under AM0 illumination.

Three parallel connected basic units create an independent basic block with separated output wiring from the solar panel. These blocks are able to generate $0.498 \mathrm{~W}$ of power under AM0 illumination conditions. Output power from two basic blocks is combined through two monolithic ideal diodes from the Linear Technology Company. Ideal diodes are placed inside of CubeSat picosatellite body due to the radiation shielding effect of picosatellite aluminum chassis. The combined power from two basic blocks of panels is processed by one switched regulator. One solar panel consists of a total four basic blocks; the rest of the two basic blocks are processed by second independent switched regulator for a higher failure tolerance. The topology of proposed solar panels is shown in Figure 7.

Such a proposed topology is fault tolerant. In case of solar cell damage, the generation of power is worsened or interrupted only in one quarter of the solar panel due to separation of the blocks by ideal diodes. In the case of switched regulator failure, the generation of power is worsened or interrupted only in one half of solar panel because the second half of solar panel has its own independent regulator.

\section{Practical Realization of Solar Panel}

During the practical implementation of a solar panel prototype, several aspects had to be considered. At first, the bonding of cells to the carrier plate must have good electric as well as thermal conductivity. The bottom layer of cells is positive electrode and also has to transfer heat from the cell. The necessity of elastic bonding is the second important aspect. The bonding layer has to absorb as much as possible 

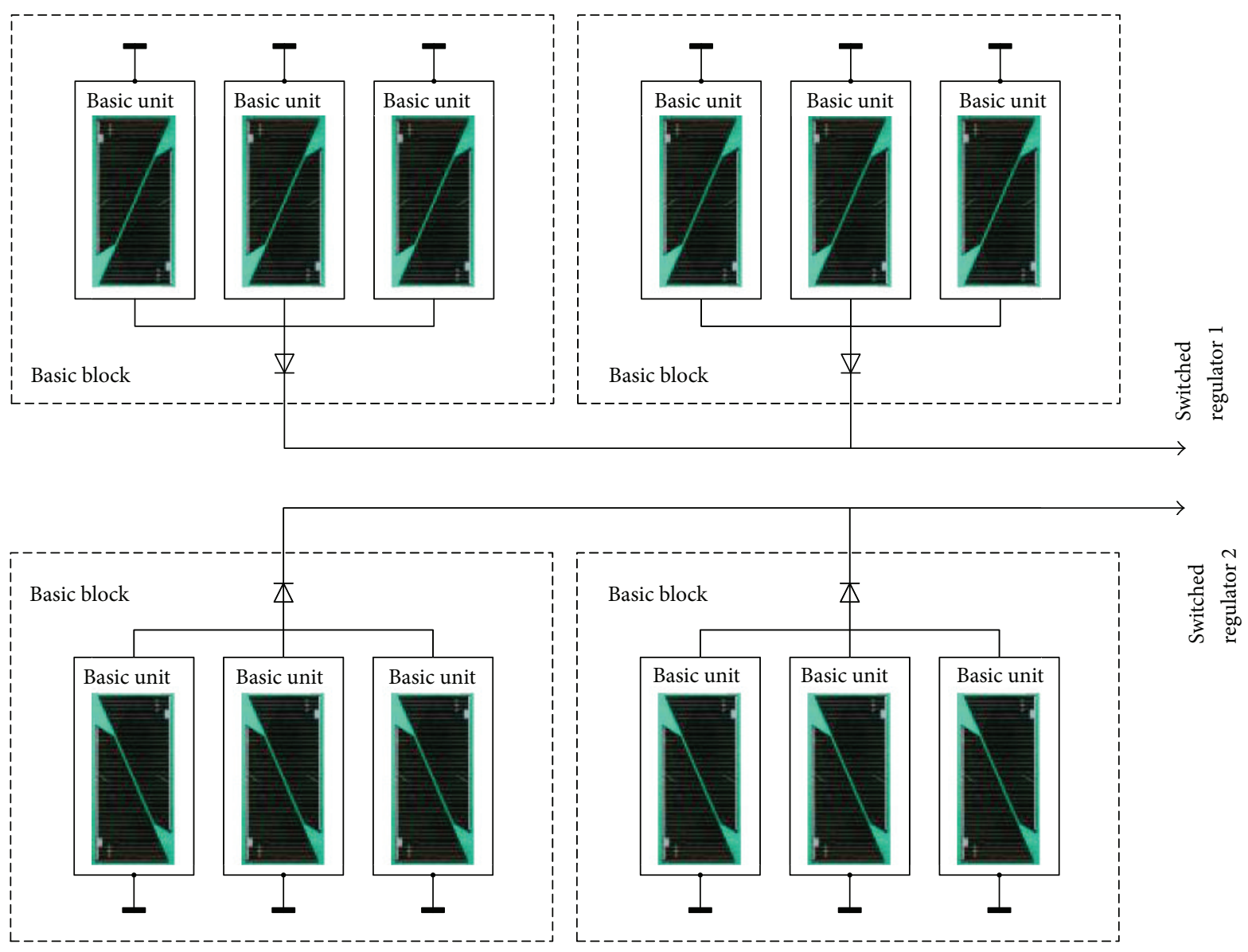

FIGURE 7: Block topology of proposed solar panel with small TASC cells.

the mechanical stress from different thermal expansion of cells and the carrier plate to avoid cell damage. From these reasons, the bonding with thermally and electrically conductive special resin was chosen for solar cell assembling.

The selection of conductive resin is not a simple task if one wants to keep the assemblage of cells manageable for small teams and the safety for cells regarding the necessary high curing temperature and to satisfy the low outgassing criteria in acceptance tests. We choose Epotek 4110-LV twocomponent epoxy resin as the bonding material due to the manageable mix ratio of components, long workability after mixing, low curing temperature, wide range of operating temperatures, and compliance with the NASA low outgassing standard.

A prototype of the proposed solar panel for the CubeSat is shown in Figure 8. The carrier plate was made from multilayer printed circuit board, where the inner layers were used for wiring of cell interconnection and also for the ladder shape design for temperature measurement of cells. The outer layer was used for creation of bondage points where the epoxy resin is applied and cells are electrically as well as thermally connected.

The solar cells have to be precisely placed on the bonding points and held in position during the curing of epoxy resin. For this purpose, we prepared a stainless template sheet which is placed over the carrier plate during the assembling and epoxy curing. The TASC solar cells are picked and moved into template sheet by simple vacuum tweezers.

The prepared prototype is suited as a deployable panel (both sides covered by cells) because the basic CubeSat walls offer a small area leading to low power generation. Assembled prototype of the deployable panel has only 22 cells on top layer and 20 cells on bottom layer; full assembling with 24 cells is expected in the future prototype.

Price of materials (solar cells and conductive resin) and cost of printed circuit board manufacturing with stainless template sheet do not exceed 350 Euro for double side coated solar panel. For comparison, commercially available CubeSat one side coated solar panel costs 2500 Euro.

\section{Solar Panel Arrangement on PilsenCUBE}

In Figure 6 it is shown that even gallium arsenide based high efficiency solar cells do not offer too much power for CubeSat supply. In long-term operation in low Earth orbits, this amount of power will decrease due to radiation damage of the cells and the power requirements of picosatellite subsystems will increase for the same reason which was discovered during radiation tests on a cobalt source. Moreover, the 


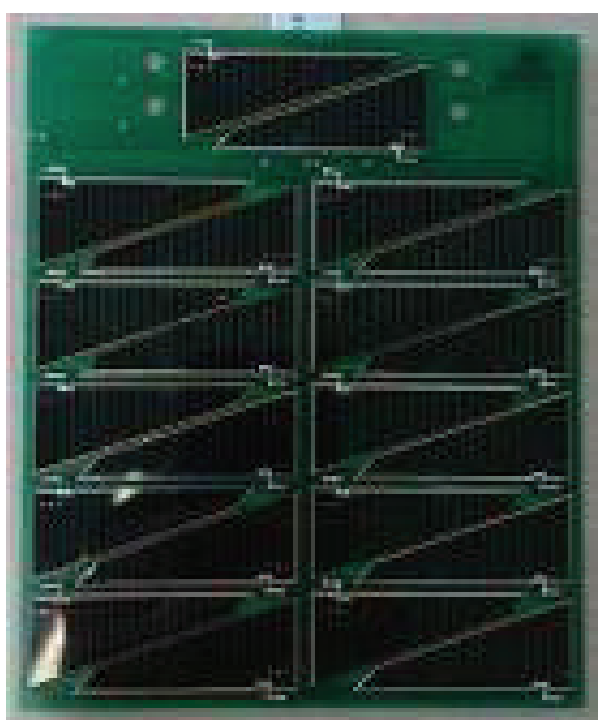

(a)

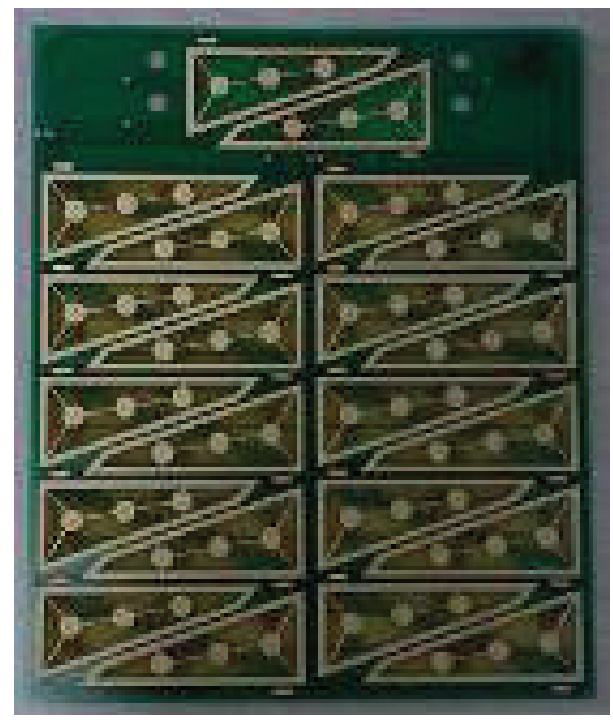

(b)

FIgURE 8: Photos of assembled and not assembled solar panel prototype for PilsenCUBE picosatellite.

efficiency of energy saving in accumulators will quickly fall with the number increase of charging-discharging cycles.

To ensure a sufficient amount of power for the onboard experiments, we propose a flower-like deployable solar panel arrangement as a complement to the basic solar panels placed on picosatellite walls. A simple deployable system for four double side coated panels uses small springs for panel deploying and a burnable cable for panels held in a closed state during the launch. Our deployable system is compatible with the dimensional requirements of a PPOD container [1], in which the CubeSat picosatellites are launched. The arrangement of the four deployable panels is shown in Figure 9.

Computations with measured efficiency dependencies for the six solid panels and four deployable double side panels were done with their own software and visualized in the same graph as previous calculations in Section 2; however, the scale of color representation had to be changed for better readability of all graphs. Each side of panels consists of 24 TASC solar cells organized into 12 basic units and 4 basic blocks. The operational temperature of the solar cells is set to $60^{\circ} \mathrm{C}$. The results of calculations are shown in Figure 10. The origin of coordinates $\left(0^{\circ}\right.$ azimuth and $0^{\circ}$ elevation) is placed in the direction of maximal power generation, for example, perpendicularly to open the flower-like shape of the panels (from top direction in Figure 9).

With deployable panels, the maximum of accessible power increases to $7.04 \mathrm{~W}$ which represents a significant improvement in comparison to $2.47 \mathrm{~W}$ without deployable panels (see Figure 6). Also, the mean power increases from $2.15 \mathrm{~W}$ to $4.55 \mathrm{~W}$ and the minimum power increases from $1.68 \mathrm{~W}$ to $2.96 \mathrm{~W}$. With the proposed deployable panels it is reasonable to try to carry out an active attitude control system for panels targeting toward the Sun. In our previous work [14], we proposed such a system with three orthogonal magnetic

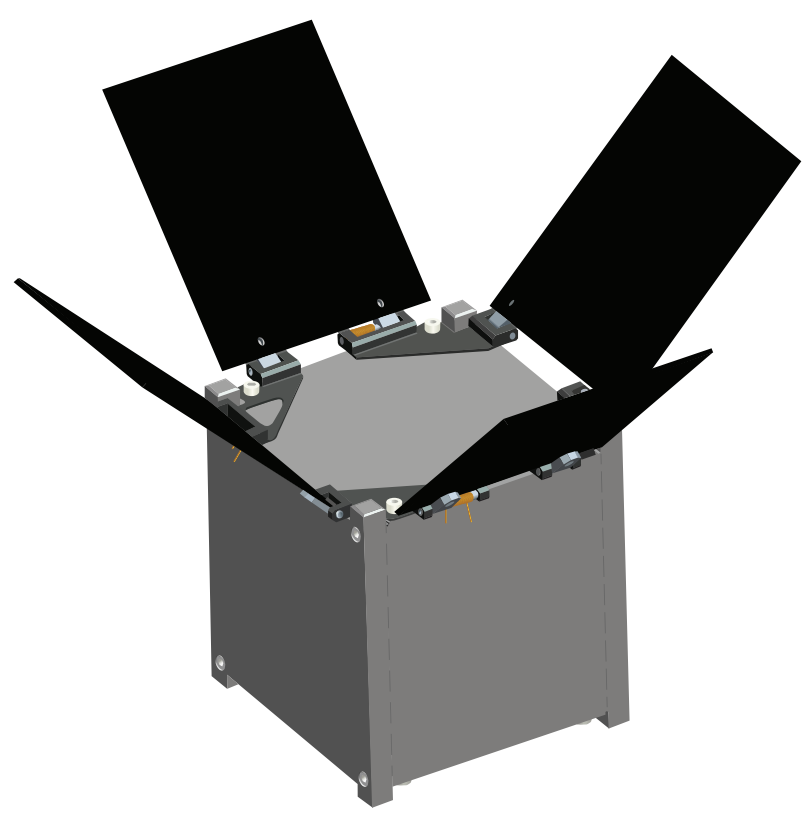

FIgUre 9: Deployable solar panels on PilsenCUBE picosatellite.

coils which are able to completely change the CubeSat attitude over several minutes with a power consumption of approximately $0.3 \mathrm{~W}$.

With asymmetric deployable system (as ours in Figure 9) also a problem with distance between centre of mass and geometrical centre of picosatellite arises. Minimizing of this distance is important for design simplification of an active attitude control system. In our project we will use allowed tolerance $2 \mathrm{~cm}$ for distance of these two points during the launch with closed solar panels. Centre of mass will be 


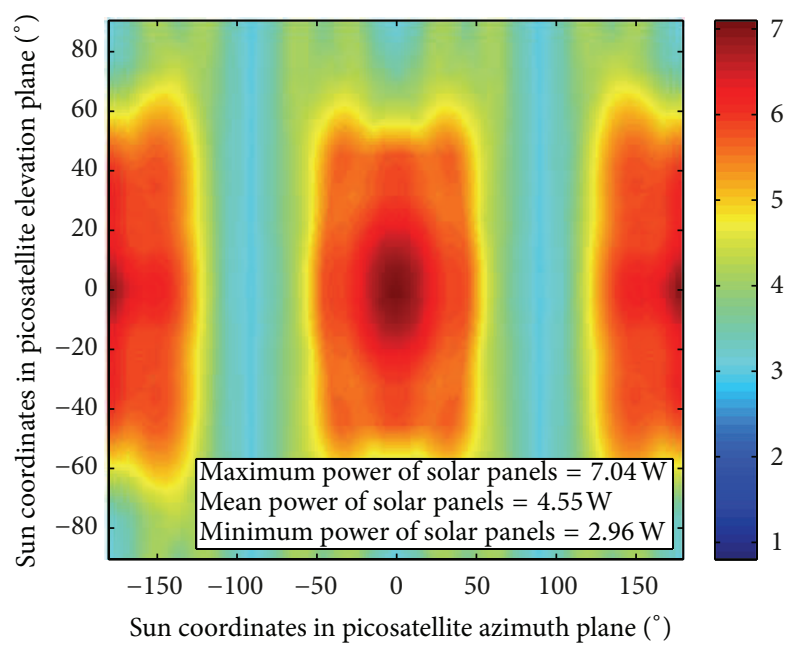

Figure 10: Power generation of gallium arsenide based solar panels placed on PilsenCUBE picosatellite including deployable panels-angle efficiency dependence is covered; $60^{\circ} \mathrm{C}$ operational temperature is considered.

projected lower (in Figure 9) than geometrical centre of picosatellite cube shape and after solar panels releasing the centre of mass it will shift toward the geometrical centre.

\section{Conclusions}

The released gallium arsenide based solar cells in the form of small area triangular strings (TASC) have opened the way for construction of CubeSat solar panels similar to large area panels for conventional satellites. In this paper, we presented a design of such panels with higher reliability in the case of electronic failures or cell damages. Our panels are also capable of being used as deployable solar panel for a power generation increase in small CubeSat picosatellites. In the case of our PilsenCUBE picosatellite, the usage of four deployable panels increases the mean value of accessible power more than 2.1 times in comparison to CubeSat without deployable panels. In the case of proper function of active attitude control system (panels are targeted toward the Sun for maximal power generation), the usage of deployable panels increases the maximal value of accessible power more than 2.8 times.

The main advantage of our solar panel solution is also in keeping assembling technology as simple as possible for construction in small teams with limited access to the highend facilities. All the designs are also compatible with the main idea of CubeSat technology, for example, to allow for low cost space missions.

In this paper, we also presented a way for more accurate calculations of accessible solar power in which the important cells parameters are not neglected as in common and often used calculations. This will help to better plan a picosatellite operation without unexpected power emergency states. We also referred to several more topics related to CubeSat picosatellite design which could be interesting for growing community of CubeSat designers at universities or private companies.

\section{Conflict of Interests}

The authors declare that there is no conflict of interests regarding the publication of this paper.

\section{Acknowledgments}

This project was funded by the Czech Science Foundation and registered as Project no. 102/09/0455: Power efficient space probe for experimental research based on picosatellite. This work was also supported by the institutional support for young researchers (POSTDOC) at the University of West Bohemia.

\section{References}

[1] S. Lee and R. Munakata, CubeSat Design Specification, revision 12, 2009, http://www.cubesat.org/images/developers/.

[2] I. Veřtát, J. Hrouda, and J. Hofman, "Spectrolab triangular solar cell evaluation for usage in PilsenCUBE Picosatellite," in Proceedings of the International Conference on Applied Electronics (AE '10), pp. 1-4, September 2010.

[3] G. Horváth, D. Czifra, G. Marosy, and Z. Várhegyi, “Thermal design and characterization of solar cell arrays aimed to be used in CubeSat missions," in Proceedings of the 18th International Workshop on Thermal Investigation of ICs and Systems (THERMINIC '12), pp. 1-4, September 2012.

[4] H. Kayal, F. Baumann, K. Briess, and S. Montenegro, "BEESAT: a pico satellite for the on orbit verification of micro wheels," in Proceedings of the 3rd International Conference on Recent Advances in Space Technologies (RAST '07), pp. 497-502, June 2007.

[5] A. M. Aly and G. Bitsuamlak, "Aerodynamics of groundmounted solar panels: test model scale effects," Journal of Wind Engineering and Industrial Aerodynamics, vol. 123, part A, pp. 250-260, 2013.

[6] A. Aly and G. Bitsuamlak, "Wind-induced pressures on solar panels mounted on residential homes," Journal of Architectural Engineering, vol. 20, no. 1, Article ID 04013003, 2014.

[7] E. Grossman, I. Gouzman, and R. Verker, "Debris/micrometeoroid impacts and synergistic effects on spacecraft materials," MRS Bulletin, vol. 35, no. 1, pp. 41-47, 2010.

[8] V. M. Smirnov, A. S. Semenov, V. G. Sokolov, V. P. Konoshenko, and I. I. Kovalyov, "Study of micrometeoroid and orbital debris effects on the solar panels retrieved from the space station “MIR'”' Space Debris, vol. 2, no. 1, pp. 1-7, 2010.

[9] J. Nilson, Optical design and characterization of solar concentrators for photovoltaics, [Licentiate thesis], Lund University, Lund, Sweden, 2005.

[10] T. M. Tritt, H. Böttner, and L. Chen, “Thermoelectrics: direct solar thermal energy conversion,” MRS Bulletin, vol. 33, no. 4, pp. 366-368, 2008.

[11] Y. Vorobiev, J. González-Hernández, P. Vorobiev, and L. Bulat, "Thermal-photovoltaic solar hybrid system for efficient solar energy conversion," Solar Energy, vol. 80, no. 2, pp. 170-176, 2006. 
[12] J. Masopust, I. Vertat, A. Vobornik et al., "PilsenCUBEPicosatellite project at the University of West Bohemia in Pilsen," in Proccedings of the 5th Pico- and Nanosatellite Workshop, Würzburg, Germany, 2011.

[13] I. Vertat, "Adaptable hybrid DM-FSK/DQPSK modulation for satellite communication," in Proceedings of the 4th European CubeSat Symposium, Karman Institute for Fluid Dynamics, Ecole Royale Militaire, Brussels, Belgium, 2012.

[14] M. Matoušů and I. Veřtát, "Design of magnetic stabilization coils for PilsenCUBE Picosatellite," in Proceedings of the International Conference on Applied Electronics (AE '11), pp. 251-254, University of West Bohemia, Pilsen, Czech Republic, September 2011. 

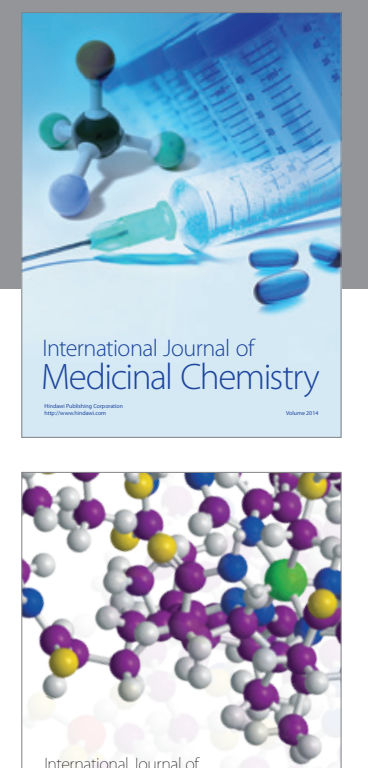

\section{Carbohydrate} Chemistry

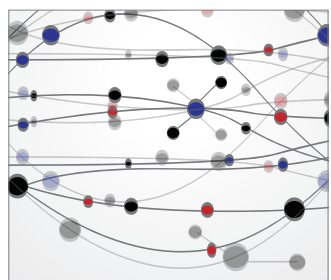

The Scientific World Journal
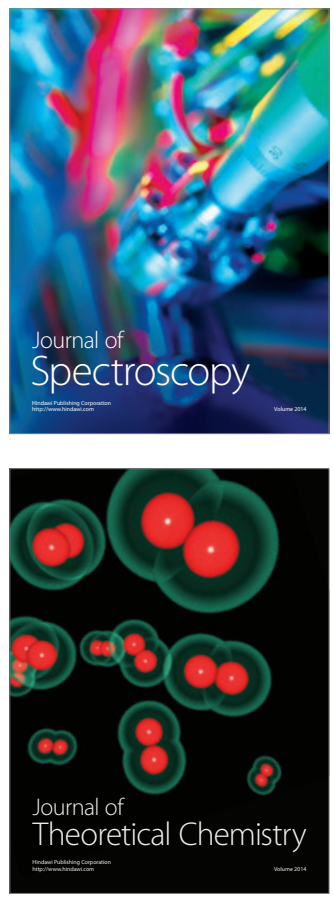
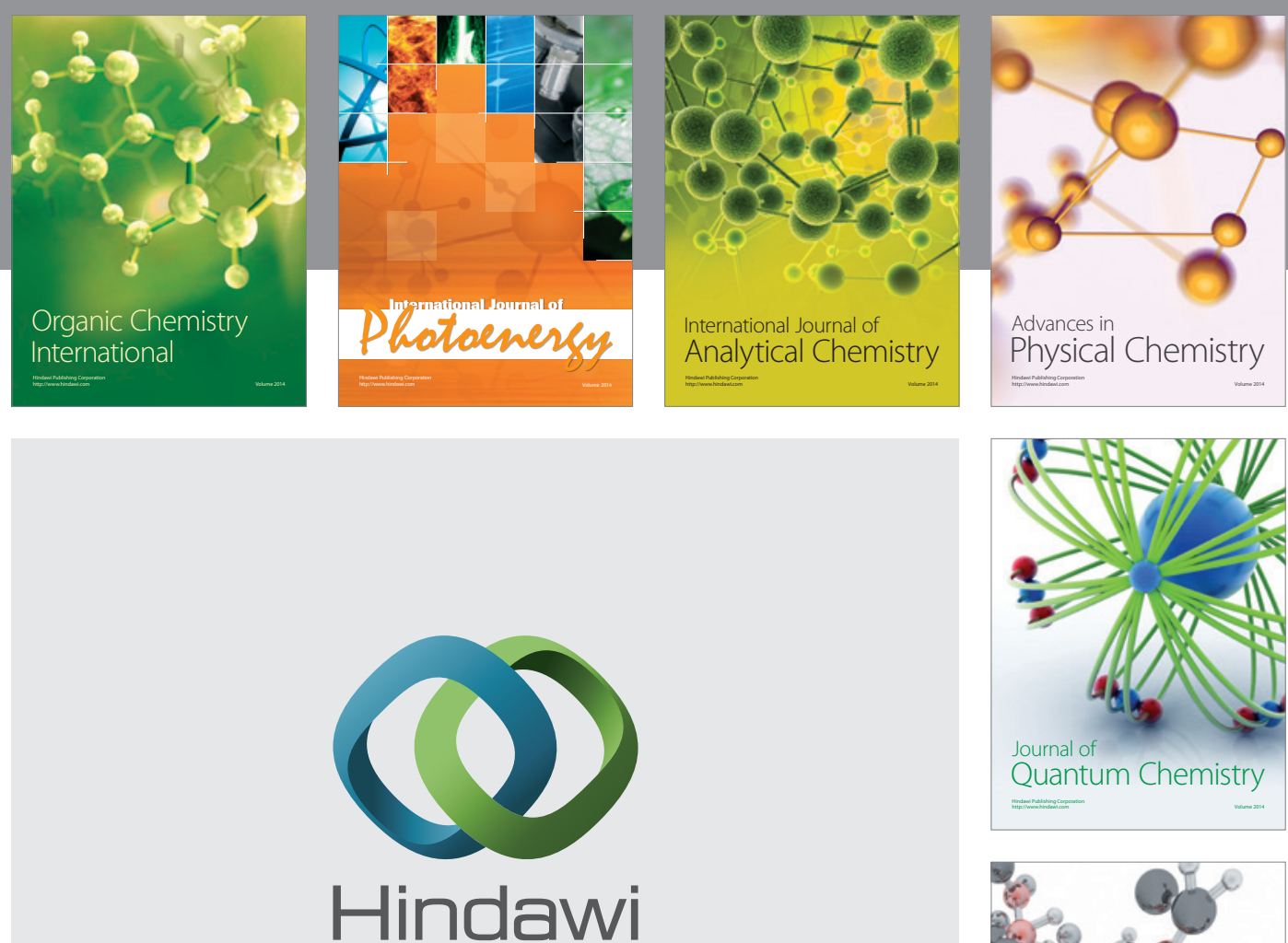

Submit your manuscripts at

http://www.hindawi.com

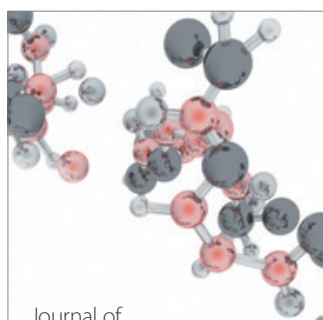

Analytical Methods

in Chemistry

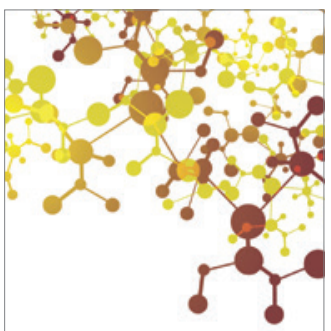

Journal of

Applied Chemistry

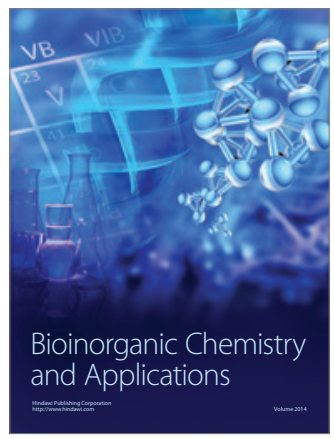

Inorganic Chemistry
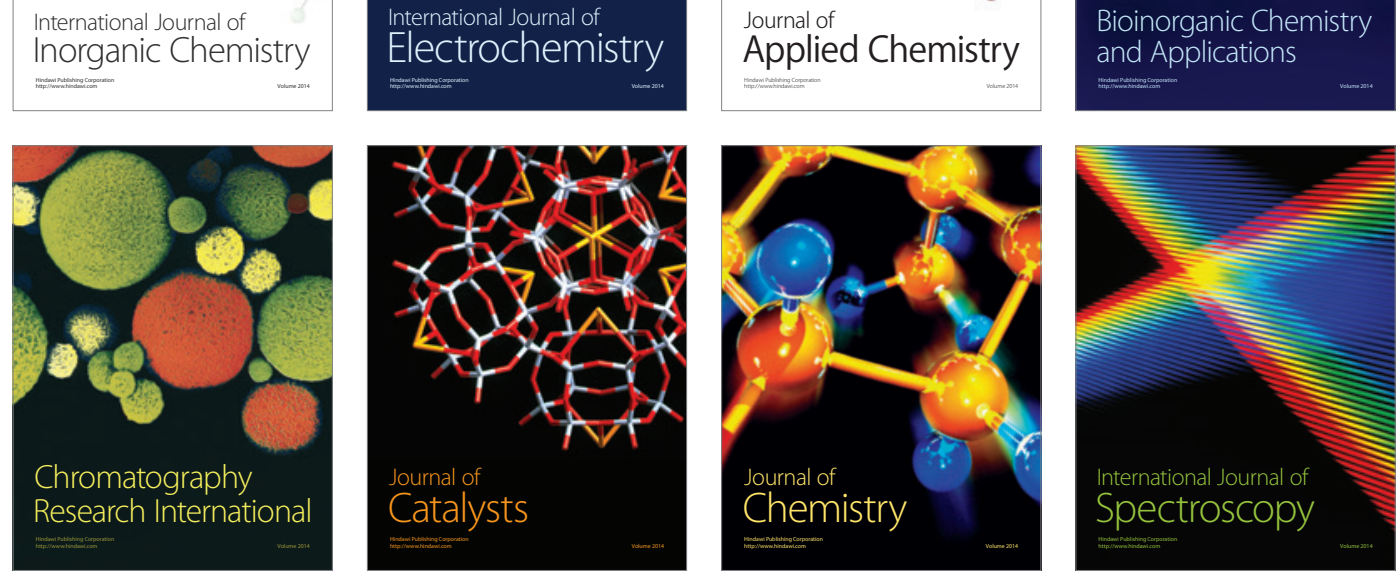